\title{
Cascade High-Gain Observers in Output Feedback Control
}

\author{
Hassan K. Khalil, \\ Department of Electrical and Computer Engineering \\ Michigan State University \\ 428 S. Shaw Lane, East Lansing, MI 48824, USA.
}

\begin{abstract}
High-gain observers proved to be a useful tool in the design of output feedback control of nonlinear systems. However, the observer faces a numerical challenge when its dimension is high. For an observer of dimension $\rho$ and a high-gain parameter $k$, the observer gain is of the order of $k^{\rho}$ and the observer variables could be of the order of $k^{\rho-1}$ during the transient period. This paper presents a new high-gain observer that is based on cascading lower-dimensional observers with saturation functions in between them. The observer gain in the new observer is of the order of $k$ and its variables are limited to be of the order of $k$ during the transient period. It is shown that the cascade observer has properties similar to the standard one. In particular, a nonlinear separation principle is proved.
\end{abstract}

Key words: High-gain observers; Nonlinear systems; Output feedback

\section{Introduction}

For over quarter of a century, high-gain observers have been used extensively in the design of output feedback control of nonlinear systems; see [6] and the references therein. They provided the first (non-local) nonlinear separation principle for nonlinear systems [3,9]. Not only does the observer recover stability achieved under state feedback, it also recovers its performance in the sense that the trajectories of the system under output feedback approach those under state feedback as the observer gain increases. The separation principle holds not only because the observer gain is made high, but also by designing the feedback control as a globally bounded function of the observer estimates to overcome the peaking phenomenon of the observer [3]. However, the standard high-gain observer faces a numerical challenge if its dimension, $\rho$, is high. The design of the observer is parameterized by a small parameter $\varepsilon$ and we refer to $k=1 / \varepsilon$ as the high-gain parameter. The observer gains are proportional to powers of $k$, with $k^{\rho}$ as the highest power. Moreover, during the transient period the internal states of the observer could peak to large values, which are proportional to powers of $k$, with $k^{\rho-1}$ as the highest

\footnotetext{
* This work was supported by the National Science Foundation under grant numbers ECCS-1128476. A preliminary version of this paper was presented at the 2016 IEEE Conference on Decision and Control, Las Vegas, NV.
}

Email address: Khalil@msu.edu (Hassan K. Khalil). power. These features pose a challenge in the numerical implementation of the observer when $\rho$ is high because in digital implementation both parameters and signals have to be represented within the finite wordlength of the digital system. It is worthwhile to note that while it is typical to saturate the state estimates or the control signal before applying the control to the plant, such saturation takes place at the output side of the observer so it does not prevent peaking in the internal variables of the observer.

Peaking of the observer's internal variables is eliminated in [7] by using estimate projection. This technique was developed to deal with a class of nonlinear systems that are not uniformly completely observable. While the technique is elegant, most results that dealt with uniformly observable systems continued to use saturation as a tool to overcome the effect of peaking, as it can be seen from the references of the survey paper [6]. Moreover, the observer of [7] still suffers from the drawback that its gain is proportional to $k^{\rho}$. On the other hand, the recent paper [1] has proposed a new high-gain observer where the observer gain is limited to be of the order of $k^{2}$. However, the internal states of that observer could still peak to $O\left(k^{\rho-1}\right)$ values. The dimension of this observer is $2(\rho-1)$ compared with dimension $\rho$ for the standard observer. The observer of [1] can be viewed as a cascade connection of $\rho-1$ second-order high-gain observers with feedback injection from one stage to the previous one. This idea motivated the cascade observer presented 
here, which eliminates the feedback injection, replaces second-order observers by first-order ones, and inserts saturation functions between the cascaded observers to limit peaking. The more recent papers [8] and [2] limited peaking in the low-power observer of [1] by using nested saturations. The cascade observer of this paper is different from the observers in [8] and [2]. Its dimension is $\rho$, compared with $2(\rho-1)$, and its design is simpler.

The paper is organized as follows. Section 2 reviews some basic knowledge about the standard observer. Section 3 presents the cascade observer and proves that the estimation errors decay to $O(\varepsilon)$ values within a time period $[0, T(\varepsilon)]$, where $\lim _{\varepsilon \rightarrow 0} T(\varepsilon)=0$. Section 4 proves a nonlinear separation theorem when the observer is used in feedback control. The results of Sections 3 and 4 are illustrated by simulation. Finally, some concluding remarks are given in Section 5 .

\section{Preliminaries}

A high-gain observer for the system

$$
\begin{aligned}
\dot{w} & =f_{0}(w, x, u) \\
\dot{x}_{i} & =x_{i+1}, \quad \text { for } 1 \leq i \leq \rho-1 \\
\dot{x}_{\rho} & =\phi(w, x, u) \\
y & =x_{1}
\end{aligned}
$$

is given by

$$
\begin{aligned}
& \dot{\hat{x}}_{i}=\hat{x}_{i+1}+\frac{\alpha_{i}}{\varepsilon^{i}}\left(y-\hat{x}_{1}\right), \text { for } 1 \leq i \leq \rho-1 \\
& \dot{\hat{x}}_{\rho}=\phi_{0}(\hat{x}, u)+\frac{\alpha_{\rho}}{\varepsilon^{\rho}}\left(y-\hat{x}_{1}\right)
\end{aligned}
$$

where $\phi_{0}$ is a nominal model of $\phi, \varepsilon$ is a sufficiently small positive constant, and $\alpha_{1}$ to $\alpha_{\rho}$ are chosen such that the roots of

$$
s^{\rho}+\alpha_{1} s^{\rho-1}+\cdots+\alpha_{\rho-1} s+\alpha_{\rho}=0
$$

have negative real parts. We assume that $f_{0}, \phi$, and $\phi_{0}$ are locally Lipschitz in their arguments and

$$
\left|\phi(w, x, u)-\phi_{0}(z, u)\right| \leq L\|x-z\|+M
$$

for all $z \in R^{\rho}$ and all bounded $w, x$, and $u$. Because

$$
\begin{aligned}
& \phi(w, x, u)-\phi_{0}(z, u)= \\
& \phi(w, x, u)-\phi_{0}(x, u)+\phi_{0}(x, u)-\phi_{0}(z, u)
\end{aligned}
$$

and $\phi_{0}(x, u)$ can be chosen to be globally Lipschitz in $x$ by saturating its $x$-argument outside a compact set, (8) requires the modeling error $\phi(w, x, u)-\phi_{0}(x, u)$ to be bounded. We can choose $\phi_{0}=0$, which would be a natural choice if no information is available on $\phi$. In this case, (8) holds with $L=0$. On the other hand, if $\phi$ is known and either it is not function of $w$ or $w$ is measured, we can take $\phi_{0}=\phi$ with the $x$-argument of $\phi$ saturated outside a compact set of interest. In this case, (8) holds with $M=0$. The estimation error $\tilde{x}_{i}=x_{i}-\hat{x}_{i}$ satisfies the inequality ${ }^{1}$

$$
\left|\tilde{x}_{i}\right| \leq \max \left\{\frac{b}{\varepsilon^{i-1}}\|\tilde{x}(0)\| e^{-a t / \varepsilon}, \varepsilon^{\rho+1-i} c M\right\}
$$

for $0<\varepsilon \leq \varepsilon^{*}$ for some positive constants $a, b, c$, and $\varepsilon^{*}$. The two terms on the right-hand side of (9) show bounds on the estimation error due to two sources. The term $\left(b / \varepsilon^{i-1}\right)\|\tilde{x}(0)\| e^{-a t / \varepsilon}$ is due to the initial estimation error $\tilde{x}(0)$ and exhibits the peaking phenomenon. It illustrates the fact that the estimation error decays rapidly to small values. In particular, given any positive constant $K$, it can be seen that

$$
\frac{b}{\varepsilon^{\rho-1}} e^{-a t / \varepsilon} \leq K \varepsilon, \forall t \geq T(\varepsilon) \stackrel{\text { def }}{=} \frac{\varepsilon}{a} \ln \left(\frac{b}{K \varepsilon^{\rho}}\right)
$$

Using l'Hôpital's rule, it can be seen that $\lim _{\varepsilon \rightarrow 0} T(\varepsilon)=$ 0 . The second term $\varepsilon^{\rho+1-i} c M$ is due to the error in modeling the function $\phi$. This error will not exist if the observer is implemented with $\phi=\phi_{0}$.

In the special case where the task is to estimate the first derivative of a signal using a linear observer, the system (1)-(4) specializes to

$$
\dot{x}_{1}=x_{2}, \quad \dot{x}_{2}=\phi\left(x_{1}, x_{2}, u\right), \quad y=x_{1}
$$

and the second-order linear observer is given by

$$
\dot{\hat{x}}_{1}=\hat{x}_{2}+\left(\alpha_{1} / \varepsilon\right)\left(y-\hat{x}_{1}\right), \dot{\hat{x}}_{2}=\left(\alpha_{2} / \varepsilon^{2}\right)\left(y-\hat{x}_{1}\right)
$$

where $\alpha_{1}, \alpha_{2}$, and $\varepsilon$ are positive constants. The observer's transfer function from $y$ to $\hat{x}_{2}$ is given by

$$
\frac{\alpha_{2} s}{(\varepsilon s)^{2}+\alpha_{1}(\varepsilon s)+\alpha_{2}}
$$

The observer gain in (12) is of the order of $1 / \varepsilon^{2}$. By scaling the observer variables as $z_{1}=\hat{x}_{1}$ and $z_{2}=\varepsilon \hat{x}_{2}$, the observer is implemented by the equations

$$
\begin{aligned}
& \dot{z}_{1}=(1 / \varepsilon)\left[z_{2}+\alpha_{1}\left(y-z_{1}\right)\right], \dot{z}_{2}=\left(\alpha_{2} / \varepsilon\right)\left(y-z_{1}\right) \\
& \hat{x}_{1}=z_{1}, \quad \hat{x}_{2}=z_{2} / \varepsilon
\end{aligned}
$$

where now the highest gain is of the order of $1 / \varepsilon$.

The first derivative can also be estimated using a reduced-order linear observer of the form

$$
\dot{z}=-(\beta / \varepsilon)(z+y), \quad \hat{x}_{2}=(\beta / \varepsilon)(z+y)
$$

\footnotetext{
1 See for example [5, Section 11.4].
} 
where $\beta$ and $\varepsilon$ are positive constants. The observer's transfer function from $y$ to $\hat{x}_{2}$ is given by

$$
\frac{\beta s}{\varepsilon s+\beta}
$$

In both observers, the estimation error satisfies the inequality

$$
\left|\tilde{x}_{2}\right| \leq \max \left\{\frac{b}{\varepsilon}\|\tilde{x}(0)\| e^{-a t / \varepsilon}, \varepsilon c M\right\}
$$

for some positive constants $a, b, c$. However, comparison of the transfer functions (13) and (17) shows that the second-order observer has low-pass filtering characteristics that filter out high-frequency noise.

\section{Cascade Observer}

In this section, we present a modified high-gain observer for the system (1)-(4) that overcomes the numerical challenges in implementing the standard high-gain observer when $\rho$ is high. We assume that $f_{0}$ and $\phi$ are locally Lipschitz in their arguments and $w(t), x(t)$, and $u(t)$ are bounded for all $t \geq 0$. What is special about the system (1)-(4) is that the states $x_{2}$ to $x_{\rho}$ are derivatives of $x_{1}$. The derivative of a signal can be estimated by a first-order or second-order linear observer as we saw in the previous section. By cascading such low-order observers we can estimate higher derivatives of the signal. We choose to estimate $x_{2}$ using a second-order observer because of its low-pass filtering characteristics, but use first-order observers to estimate $x_{3}$ to $x_{\rho}$ so that the dimension of the cascade connection is $\rho$, as in the standard observer. The cascade connection is given by

$$
\begin{aligned}
\dot{z}_{1} & =(1 / \varepsilon)\left[z_{2}+\beta_{1}\left(y-z_{1}\right)\right] \\
\dot{z}_{2} & =\left(\beta_{2} / \varepsilon\right)\left(y-z_{1}\right) \\
\hat{x}_{1} & =z_{1}, \quad \hat{x}_{2}=z_{2} / \varepsilon \\
\dot{z}_{i} & =-\left(\beta_{i} / \varepsilon\right)\left(z_{i}+\hat{x}_{i-1}\right), \quad \text { for } 3 \leq i \leq \rho \\
\hat{x}_{i} & =\left(\beta_{i} / \varepsilon\right)\left(z_{i}+\hat{x}_{i-1}\right), \quad \text { for } 3 \leq i \leq \rho
\end{aligned}
$$

where $\beta_{1}$ to $\beta_{\rho}$ are positive constants. A block diagram representation of the cascade connection, in terms of the transfer functions of its stages, is shown in Figure 1. The highest gain in this observer is of the order of $1 / \varepsilon$, but the estimate of the highest derivative could be of the order of $1 / \varepsilon^{\rho-1}$ during the peaking period. This peaking can be reduced by saturating the estimates. If $x(t)$ belongs to a compact set $X$, take $M_{i}>\max _{x \in X}\left|x_{i}\right|$, for $2 \leq i \leq \rho$. The estimate of $x_{i}$ is saturated at $\pm M_{i}$. The cascade observer with saturation is given by the following equations and its block diagram representation is shown in Figure 2.

$$
\begin{aligned}
& \dot{z}_{1}=(1 / \varepsilon)\left[z_{2}+\beta_{1}\left(y-z_{1}\right)\right] \\
& \dot{z}_{2}=\left(\beta_{2} / \varepsilon\right)\left(y-z_{1}\right) \\
& \hat{x}_{1}=z_{1}, \quad \hat{x}_{2}=M_{2} \operatorname{sat}\left(\frac{z_{2}}{\varepsilon M_{2}}\right) \\
& \dot{z}_{i}=-\left(\beta_{i} / \varepsilon\right)\left(z_{i}+\hat{x}_{i-1}\right) \\
& \hat{x}_{i}=M_{i} \operatorname{sat}\left(\frac{\beta_{i}\left(z_{i}+\hat{x}_{i-1}\right)}{\varepsilon M_{i}}\right)
\end{aligned}
$$

where $\operatorname{sat}(y)=\min \{|y|, 1\} \operatorname{sign}(y)$ and (26) and (27) hold for $3 \leq i \leq \rho$. In the first stage, the signal $z_{2} / \varepsilon$ could be of the order of $1 / \varepsilon$ but the estimate $\hat{x}_{2}$ is bounded uniformly in $\varepsilon$. For all the other stages, the deriving input $\hat{x}_{i-1}$ is bounded uniformly in $\varepsilon$, and so is $z_{i}$. Therefore, $\left(\beta_{i} / \varepsilon\right)\left(z_{i}+\hat{x}_{i-1}\right)$ is limited to be of the order of $1 / \varepsilon$.

Theorem 1 Under the stated assumptions, there is $\varepsilon^{*}>$ 0 such that for $0<\varepsilon \leq \varepsilon^{*}$, the estimation errors $\tilde{x}_{i}=$ $x_{i}-\hat{x}_{i}$, for $1 \leq i \leq \rho$, of the cascade high-gain observer (23)-(27) are bounded and there is time $T(\varepsilon)$, with $\lim _{\varepsilon \rightarrow 0} T(\varepsilon)=0$, such that

$$
\begin{aligned}
& \left|\tilde{x}_{1}(t)\right| \leq a \varepsilon^{2} \\
& \left|\tilde{x}_{i}(t)\right| \leq a \varepsilon, \quad \text { for } 2 \leq i \leq \rho
\end{aligned}
$$

for all $t \geq T(\varepsilon)$, for some positive constant $a$.

Proof: Since $y$ is bounded by assumption and each stage of the observer is an exponentially stable system, the observer variables $z_{1}$ to $z_{\rho}$ are bounded. Let

$$
\xi_{1}=z_{1}, \xi_{2}=z_{2} / \varepsilon, \xi_{i}=\left(\beta_{i} / \varepsilon\right)\left(z_{i}+\xi_{i-1}\right), \text { for } 3 \leq i \leq \rho
$$

and

$$
\eta_{i}=\varepsilon^{i-1}\left(x_{i}-\xi_{i}\right), \quad \text { for } 1 \leq i \leq \rho
$$

When $\hat{x}_{2}, \ldots, \hat{x}_{i}$ are not saturated, they are equal to $\xi_{2}, \ldots, \xi_{i}$, respectively. In this case, $\eta_{2}, \ldots, \eta_{i}$ are scaled estimation errors and $\dot{z}_{k}=-\xi_{k}$ for $3 \leq k \leq \rho$. Our analysis of the estimation error will proceed stage by stage starting from the second-order observer. In each step it is shown that after a short time period the estimation error will be small and the estimate will not be saturated. For the first stage, it can be shown that $\psi_{2}=\operatorname{col}\left(\eta_{1}, \eta_{2}\right)$ satisfies the equation

$$
\varepsilon \dot{\psi}_{2}=F_{2} \psi_{2}+\varepsilon^{2} G_{23} x_{3}
$$

where

$$
F_{2}=\left[\begin{array}{ll}
-\beta_{1} & 1 \\
-\beta_{2} & 0
\end{array}\right] \text { and } G_{23}=\left[\begin{array}{l}
0 \\
1
\end{array}\right]
$$

The matrix $F_{2}$ is Hurwiz. Since $x_{3}(t)$ is bounded uniformly in $\varepsilon$, it can be shown that there is $T_{2}(\varepsilon)>0$, with $\lim _{\varepsilon \rightarrow 0} T_{2}(\varepsilon)=0$, such that $\left\|\psi_{2}(t)\right\| \leq k_{1} \varepsilon^{2}$ for all 


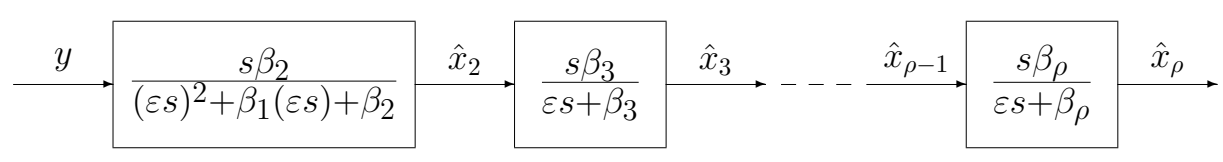

Fig. 1. Cascade realization of high-gain observer.

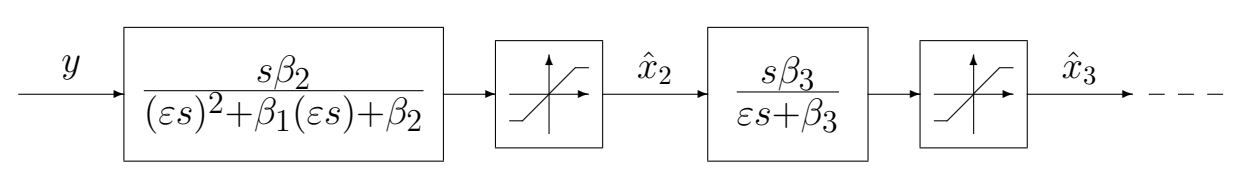

Fig. 2. Cascade realization of high-gain observer with saturation.

$t \geq T_{2}$, for some constant $k_{1} \cdot{ }^{2}$ In view of (31), we have

$$
\left|x_{1}-\xi_{1}\right| \leq k_{1} \varepsilon^{2} \quad \text { and } \quad\left|x_{2}-\xi_{2}\right| \leq k_{1} \varepsilon, \quad \forall t \geq T_{2}
$$

We conclude that, for sufficiently small $\varepsilon,\left|\xi_{2}\right| \leq M_{2}$. Therefore $\hat{x}_{i}=\xi_{i}$, for $i=1,2$, which proves (28) and the case $i=2$ of $(29)$.

We proceed to the next stage where, for $t \geq T_{2}, \eta_{3}$ satisfies the equation

$$
\begin{aligned}
\varepsilon \dot{\eta}_{3} & =\varepsilon^{3}\left(\dot{x}_{3}-\dot{\xi}_{3}\right)=\varepsilon^{3}\left[\dot{x}_{3}-\left(\beta_{3} / \varepsilon\right) \dot{z}_{3}-\left(\beta_{3} / \varepsilon^{2}\right) \dot{z}_{2}\right] \\
& =\varepsilon^{3}\left[x_{4}+\left(\beta_{3} / \varepsilon\right) \xi_{3}-\left(\beta_{2} \beta_{3} / \varepsilon^{3}\right)\left(x_{1}-z_{1}\right)\right] \\
& =-\beta_{2} \beta_{3} \eta_{1}-\beta_{3} \eta_{3}+\varepsilon^{2} \beta_{3} x_{3}+\varepsilon^{3} x_{4}
\end{aligned}
$$

This equation can be used to show an ultimate bound on $\left|\eta_{3}\right|$ of the form $k_{2} \varepsilon^{2}$. This bound and (31) show that $\left|x_{3}-\xi_{3}\right| \leq k_{2}$, which does not yield (29). We need a sharper bound on $\left|\eta_{3}\right|$ that takes the form $k_{3} \varepsilon^{3}$. Towards that end we note that $\eta_{3}$ is the output of the system

$$
\varepsilon \dot{\psi}_{3}=F_{3} \psi_{3}+\varepsilon^{2} G_{33} x_{3}+\varepsilon^{3} G_{34} x_{4}, \quad \eta_{3}=H_{3} \psi_{3}
$$

where

$$
\begin{gathered}
\psi_{3}=\left[\begin{array}{l}
\eta_{1} \\
\eta_{2} \\
\eta_{3}
\end{array}\right], \quad F_{3}=\left[\begin{array}{ccc}
-\beta_{1} & 1 & 0 \\
-\beta_{2} & 0 & 0 \\
-\beta_{2} \beta_{3} & 0 & -\beta_{3}
\end{array}\right], \quad G_{33}=\left[\begin{array}{c}
0 \\
1 \\
\beta_{3}
\end{array}\right] \\
G_{34}=\left[\begin{array}{l}
0 \\
0 \\
1
\end{array}\right], \text { and } H_{3}=\left[\begin{array}{lll}
0 & 0 & 1
\end{array}\right]
\end{gathered}
$$

The matrix $F_{3}$ is Hurwitz. To eliminate the $\varepsilon^{2} x_{3}$ term on the right-hand side of the $\dot{\psi}_{3}$-equation, we apply the

\footnotetext{
2 Throughout the proof, $k_{i}$ denotes a positive constant independent of $\varepsilon$.
}

transformation

$$
\tilde{\psi}_{3}=\psi_{3}+\varepsilon^{2} F_{3}^{-1} G_{33} x_{3}
$$

which yields

$$
\varepsilon \dot{\tilde{\psi}}_{3}=F_{3} \tilde{\psi}_{3}+\varepsilon^{3}\left(F_{3}^{-1} G_{33}+G_{34}\right) x_{4}
$$

and $\eta_{3}$ is given by

$$
\eta_{3}=H_{3} \tilde{\psi}_{3}-\varepsilon^{2} H_{3} F_{3}^{-1} G_{33} x_{3}
$$

The second term of the foregoing expression vanishes because

$$
H_{3} F_{3}^{-1} G_{33}=\left[\begin{array}{lll}
0 & 0 & 1
\end{array}\right]\left[\begin{array}{ccc}
0 & -1 / \beta_{2} & 0 \\
1 & -\beta_{1} / \beta_{2} & 0 \\
0 & 1 & -1 / \beta_{3}
\end{array}\right]\left[\begin{array}{c}
0 \\
1 \\
\beta_{3}
\end{array}\right]=0
$$

Thus, $\eta_{3}$ is the output of the system

$$
\varepsilon \dot{\tilde{\psi}}_{3}=F_{3} \tilde{\psi}_{3}+\varepsilon^{3}\left(F_{3}^{-1} G_{33}+G_{34}\right) x_{4}, \quad \eta_{3}=H_{3} \tilde{\psi}_{3}
$$

which shows that there exists $T_{3}(\varepsilon)>T_{2}(\varepsilon)$, with $\lim _{\varepsilon \rightarrow 0} T_{3}(\varepsilon)=0$, such that $\left|\eta_{3}(t)\right| \leq k_{3} \varepsilon^{3}, \forall t \geq T_{3}$, for some constant $k_{3}$. In view of (31), we have $\left|x_{3}-\xi_{3}\right| \leq k_{3} \varepsilon, \forall t \geq T_{3}$. We conclude that, for sufficiently small $\varepsilon,\left|\xi_{3}\right| \leq M_{3}$. Therefore $\hat{x}_{3}=\xi_{3}$, which proves the case $i=3$ of (29). 
Proceeding to the next stage, we note that for $t \geq T_{3}$, $\eta_{4}$ satisfies the equation

$$
\begin{aligned}
\varepsilon \dot{\eta}_{4}= & \varepsilon^{4}\left(\dot{x}_{4}-\dot{\xi}_{4}\right)=\varepsilon^{4}\left[\dot{x}_{4}-\left(\beta_{4} / \varepsilon\right) \dot{z}_{4}-\left(\beta_{4} / \varepsilon\right) \dot{\xi}_{3}\right] \\
= & \varepsilon^{3} \beta_{4} \xi_{4}-\varepsilon^{3} \beta_{4} \dot{\xi}_{3}+\varepsilon^{4} x_{5} \\
= & -\beta_{4}\left(\eta_{4}-\varepsilon^{3} x_{4}\right)+\beta_{4}\left(\varepsilon \dot{\eta}_{3}-\varepsilon^{3} \dot{x}_{3}\right)+\varepsilon^{4} x_{5} \\
= & \beta_{4} \varepsilon \dot{\eta}_{3}-\beta_{4} \eta_{4}+\varepsilon^{4} x_{5} \\
= & \beta_{4} H_{3}\left(F_{3} \psi_{3}+\varepsilon^{2} G_{33} x_{3}+\varepsilon^{3} G_{34} x_{4}\right)-\beta_{4} \eta_{4}+\varepsilon^{4} x_{5} \\
= & -\beta_{2} \beta_{3} \beta_{4} \eta_{1}-\beta_{3} \beta_{4} \eta_{3}-\beta_{4} \eta_{4}+\varepsilon^{2} \beta_{3} \beta_{4} x_{3} \\
& +\varepsilon^{3} \beta_{4} x_{4}+\varepsilon^{4} x_{5}
\end{aligned}
$$

Thus, $\eta_{4}$ is the output of the system

$\varepsilon \dot{\psi}_{4}=F_{4} \psi_{4}+\varepsilon^{2} G_{43} x_{3}+\varepsilon^{3} G_{44} x_{4}+\varepsilon^{4} G_{45} x_{5}, \eta_{4}=H_{4} \psi_{4}$

where

$$
\begin{gathered}
\psi_{4}=\left[\begin{array}{l}
\eta_{1} \\
\eta_{2} \\
\eta_{3} \\
\eta_{4}
\end{array}\right], \quad F_{4}=\left[\begin{array}{cccc}
-\beta_{1} & 1 & 0 & 0 \\
-\beta_{2} & 0 & 0 & 0 \\
-\beta_{2} \beta_{3} & 0 & -\beta_{3} & 0 \\
-\beta_{2} \beta_{3} \beta_{4} & 0 & -\beta_{3} \beta_{4} & -\beta_{4}
\end{array}\right] \\
G_{43}=\left[\begin{array}{c}
0 \\
1 \\
\beta_{3} \\
\beta_{3} \beta_{4}
\end{array}\right], \quad G_{44}=\left[\begin{array}{l}
0 \\
0 \\
1 \\
\beta_{4}
\end{array}\right], \quad G_{45}=\left[\begin{array}{l}
0 \\
0 \\
0 \\
1
\end{array}\right]
\end{gathered}
$$

and $H_{4}=\left[\begin{array}{llll}0 & 0 & 0 & 1\end{array}\right]$. The matrix $F_{4}$ is Hurwitz. We pay attention to the $\varepsilon^{2} x_{3}$ and $\varepsilon^{3} x_{4}$ terms on the right-hand side of the $\dot{\psi}_{4}$-equation because the power of $\varepsilon$ in these two terms is less than 4 . We apply two transformation to eliminate these terms. The first transformation eliminates the $\varepsilon^{2}$ term and the second one eliminates the $\varepsilon^{3}$ term. Then we combine the two transformation into one. The transformation

$$
\hat{\psi}_{4}=\psi_{4}+\varepsilon^{2} F_{4}^{-1} G_{43} x_{3}
$$

results in

$$
\varepsilon \dot{\hat{\psi}}_{4}=F_{4} \hat{\psi}_{4}+\varepsilon^{3}\left(F_{4}^{-1} G_{43}+G_{44}\right) x_{4}+\varepsilon^{4} G_{45} x_{5}
$$

Then, the transformation

$$
\tilde{\psi}_{4}=\hat{\psi}_{4}+\varepsilon^{3} F_{4}^{-1}\left(F_{4}^{-1} G_{43}+G_{44}\right) x_{4}
$$

yields

$$
\varepsilon \dot{\tilde{\psi}}_{4}=F_{4} \tilde{\psi}_{4}+\varepsilon^{4}\left(F_{4}^{-2} G_{43}+F_{4}^{-1} G_{44}+G_{45}\right) x_{5}
$$

The overall transformation is

$$
\tilde{\psi}_{4}=\psi_{4}+\varepsilon^{2} F_{4}^{-1} G_{43} x_{3}+\varepsilon^{3} F_{4}^{-1}\left(F_{4}^{-1} G_{43}+G_{44}\right) x_{4}
$$

and

$$
\begin{aligned}
\eta_{4}= & H_{4} \tilde{\psi}_{4}-\varepsilon^{2} H_{4} F_{4}^{-1} G_{43} x_{3}-\varepsilon^{3} H_{4} F_{4}^{-2} G_{43} x_{4} \\
& -\varepsilon^{3} H_{4} F_{4}^{-1} G_{44} x_{4}
\end{aligned}
$$

It can be verified that

$$
H_{4} F_{4}^{-1} G_{43}=0, H_{4} F_{4}^{-2} G_{43}=0 \text {, and } H_{4} F_{4}^{-1} G_{44}=0
$$

Thus, $\eta_{4}$ is the output of the system

$\varepsilon \dot{\tilde{\psi}}_{4}=F_{4} \tilde{\psi}_{4}+\varepsilon^{4}\left(F_{4}^{-2} G_{43}+F_{4}^{-1} G_{44}+G_{45}\right) x_{5}, \eta_{4}=H_{4} \tilde{\psi}_{4}$

and there exists $T_{4}(\varepsilon)>T_{3}(\varepsilon)$, with $\lim _{\varepsilon \rightarrow 0} T_{4}(\varepsilon)=0$, such that $\left|\eta_{4}(t)\right| \leq k_{4} \varepsilon^{4}, \forall t \geq T_{4}$, for some constant $k_{4}$. In view of (31), we have $\left|x_{4}-\xi_{4}\right| \leq k_{4} \varepsilon, \forall t \geq T_{4}$. We conclude that, for sufficiently small $\varepsilon,\left|\xi_{4}\right| \leq M_{4}$. Therefore $\hat{x}_{4}=\xi_{4}$, which proves the case $i=4$ of $(29)$. Proceeding in the same way to the next stages, consider the stage $i>4$ where, for $t \geq T_{i-1}(\varepsilon)$, we have

$$
\begin{aligned}
\varepsilon \dot{\eta}_{i} & =\varepsilon^{i}\left(\dot{x}_{i}-\dot{\xi}_{i}\right)=\varepsilon^{i}\left[\dot{x}_{i}-\left(\beta_{i} / \varepsilon\right) \dot{z}_{i}-\left(\beta_{i} / \varepsilon\right) \dot{\xi}_{i-1}\right] \\
& =\varepsilon^{i-1} \beta_{i} \xi_{i}-\varepsilon^{i-1} \beta_{i} \dot{\xi}_{i-1}+\varepsilon^{i} x_{i+1} \\
& =-\beta_{i}\left(\eta_{i}-\varepsilon^{i-1} x_{i}\right)+\beta_{i}\left(\varepsilon \dot{\eta}_{i-1}-\varepsilon^{i-1} \dot{x}_{i-1}\right)+\varepsilon^{i} x_{i+1} \\
& =\beta_{i} \varepsilon \dot{\eta}_{i-1}-\beta_{i} \eta_{i}+\varepsilon^{i} x_{i+1}
\end{aligned}
$$

Thus, $\eta_{i}$ is the the output of the system

$$
\varepsilon \dot{\psi}_{i}=F_{i} \psi_{i}+\sum_{k=3}^{i} G_{i k} \varepsilon^{k-1} x_{k}+G_{i, i+1} \varepsilon^{i} x_{i+1}, \eta_{i}=H_{i} \psi_{i}
$$

where

$$
\psi_{i}=\left[\begin{array}{c}
\psi_{i-1} \\
\eta_{i}
\end{array}\right], \quad F_{i}=\left[\begin{array}{cc}
F_{i-1} & 0 \\
\beta_{i} H_{i-1} F_{i-1} & -\beta_{i}
\end{array}\right]
$$

$$
G_{i k}=\left[\begin{array}{c}
G_{i-1, k} \\
\beta_{i} H_{i-1} G_{i-1, k}
\end{array}\right], \quad G_{i, i+1}=H_{i}^{T}
$$

and $H_{i}=\left[\begin{array}{llll}0 & \ldots & 0 & 1\end{array}\right]_{1 \times i}$. When $i=\rho$, the $x_{i+1}$ term in the $\dot{\psi}_{\rho}$-equation is replaced by $\phi$. From the block triangular structure of $F_{i}$ and the fact that $F_{4}$ is Hurwitz, it is clear that the matrices $F_{5}, F_{6}, \ldots, F_{\rho}$ are all Hurwitz.

Claim: For $3 \leq i \leq \rho$,

$$
H_{i} F_{i}^{-j} G_{i k}=0, \text { for } 1 \leq j \leq i-k+1,3 \leq k \leq i
$$


Proof of the claim: We have already shown (35) for $i=3$. We prove it for $i=4,5,6, \ldots, \rho$ by mathematical induction. Suppose it is true for $i-1$ and show it for $i$.

$$
\begin{aligned}
F_{i}^{-1} G_{i k} & =\left[\begin{array}{cc}
F_{i-1}^{-1} & 0 \\
H_{i-1} & -1 / \beta_{i}
\end{array}\right]\left[\begin{array}{c}
G_{i-1, k} \\
\beta_{i} H_{i-1} G_{i-1, k}
\end{array}\right] \\
& =\left[\begin{array}{c}
F_{i-1}^{-1} G_{i-1, k} \\
0
\end{array}\right] \\
F_{i}^{-2} G_{i k} & =\left[\begin{array}{cc}
F_{i-1}^{-1} & 0 \\
H_{i-1} & -1 / \beta_{i}
\end{array}\right]\left[\begin{array}{c}
F_{i-1}^{-1} G_{i-1, k} \\
0
\end{array}\right] \\
& =\left[\begin{array}{c}
F_{i-1}^{-2} G_{i-1, k} \\
0
\end{array}\right] \\
\vdots & \left.=\vdots \begin{array}{c}
F_{i-1}^{-(i-k+1)} G_{i-1, k} \\
0
\end{array}\right] \\
F_{i}^{-(i-k+1)} G_{i k} & =\left[\begin{array}{c}
0
\end{array}\right]
\end{aligned}
$$

Pre-multiplication by $H_{i}$ completes the proof of the claim.

Applying the transformation

$$
\tilde{\psi}_{i}=\psi_{i}+\sum_{k=3}^{i} \sum_{j=1}^{i-k+1} F_{i}^{-j} G_{i k} \varepsilon^{k+j-2} x_{k+j-1}
$$

to (34) and using (35) show, after some lengthy but straightforward manipulation, that $\eta_{i}$ is the output of the system

$$
\varepsilon \dot{\tilde{\psi}}_{i}=F_{i} \tilde{\psi}_{i}+\varepsilon^{i} \sum_{k=3}^{i+1} F_{i}^{-(i-k+1)} G_{i k} x_{i+1}, \quad \eta_{i}=H_{i} \tilde{\psi}_{i}
$$

Therefore, there exists $T_{i}(\varepsilon)$, with $\lim _{\varepsilon \rightarrow 0} T_{i}(\varepsilon)=0$, such that $\left|\eta_{i}(t)\right| \leq k_{i} \varepsilon^{i}, \forall t \geq T_{i}$, for some constant $k_{i}$. In view of $(31)$, we have $\left|x_{i}-\xi_{i}\right| \leq k_{i} \varepsilon, \forall t \geq T_{i}$. which completes the proof of the theorem.

While the cascade high-gain observer (23)-(27) has a numerical advantage over the standard high-gain observer (5)-(6) when $\rho$ is high, it has some drawbacks as well. First, if the standard high-gain observer is implemented with $\phi_{0}=\phi$, the estimation error will converge to zero as time tends to infinity. This can be seen from (9) because in this case $M=0$. Such convergence will not take place in the cascade observer, which does not use the function $\phi$. Second, the ultimate bound on the estimation error in the case of the standard high-gain observer is seen from (9) to be $\left|\tilde{x}_{i}\right| \leq \varepsilon^{\rho+1-i} c M$. When contrasted with the ultimate bound in the cascade high-gain observer, given by (28) and (29), we see that, except for the bound on $\left|x_{\rho}-\hat{x}_{\rho}\right|$, the ultimate bound is orders of magnitude smaller in the case of the standard observer. This point is illustrated further by simulation in Example 1.

Example 1 We use the standard and cascade high-gain observers to estimate the states of the system

$$
\dot{x}_{1}=x_{2}, \quad \dot{x}_{2}=x_{3}, \quad \dot{x}_{3}=x_{4}, \quad \dot{x}_{4}=\phi(x), \quad y=x_{1}
$$

where

$$
\begin{aligned}
\phi(x)= & -\left(x_{1}+2 x_{2}+2 x_{3}+2 x_{4}\right) \\
& +\left[1-\left(x_{1}+2 x_{2}+x_{3}\right)^{2}\right]\left(x_{2}+2 x_{3}+x_{4}\right)
\end{aligned}
$$

The system is constructed by cascading a van der Pol equation with a second-order exponentially stable linear system; then transforming the whole system into the normal form. Therefore, the solution $x(t)$ is periodic. It can be seen by simulation that for all initial states in the set $\left\{\left|x_{i}\right| \leq 1, i=1,2,3,4\right\}$, the solution satisfies $\left|x_{2}(t)\right| \leq 1.7,\left|x_{3}(t)\right| \leq 1.2$, and $\left|x_{4}(t)\right| \leq 2.7$. Therefore, we choose the saturation levels for the estimates $\hat{x}_{2}, \hat{x}_{3}$, and $\hat{x}_{4}$ as $\pm 2, \pm 1.5$, and \pm 3 , respectively. For the two observers, we assign all eigenvalues at $-1 / \varepsilon$. The standard observer is designed as a linear one by taking the function $\phi_{0}$ of (6) to be zero. It is given by

$$
\begin{aligned}
& \dot{z}_{1}=z_{2}+(4 / \varepsilon)\left(y-z_{1}\right), \quad \hat{x}_{1}=z_{1} \\
& \dot{z}_{2}=z_{3}+\left(6 / \varepsilon^{2}\right)\left(y-z_{1}\right), \quad \hat{x}_{2}=z_{2} \\
& \dot{z}_{3}=z_{4}+\left(4 / \varepsilon^{3}\right)\left(y-z_{1}\right), \quad \hat{x}_{3}=z_{3} \\
& \dot{z}_{4}=\left(1 / \varepsilon^{4}\right)\left(y-z_{1}\right), \quad \hat{x}_{4}=z_{4}
\end{aligned}
$$

The cascade observer is given by

$$
\begin{aligned}
& \dot{z}_{1}=(1 / \varepsilon)\left[z_{2}+2\left(y-z_{1}\right)\right], \quad \hat{x}_{1}=z_{1} \\
& \dot{z}_{2}=(1 / \varepsilon)\left(y-z_{1}\right), \quad \hat{x}_{2}=2 \operatorname{sat}\left(z_{2} /(2 \varepsilon)\right) \\
& \dot{z}_{3}=-(1 / \varepsilon)\left(z_{3}+\hat{x}_{2}\right), \quad \hat{x}_{3}=1.5 \operatorname{sat}\left(\left(z_{3}+\hat{x}_{2}\right) /(1.5 \varepsilon)\right) \\
& \dot{z}_{4}=-(1 / \varepsilon)\left(z_{4}+\hat{x}_{3}\right), \quad \hat{x}_{4}=3 \operatorname{sat}\left(\left(z_{4}+\hat{x}_{3}\right) /(3 \varepsilon)\right)
\end{aligned}
$$

Simulation results are shown in Figures 3 to 6 for $\varepsilon=$ $0.001, x_{i}(0)=1$ for $i=1,2,3,4$, and zero initial conditions for the observers. Figure 3 shows the states $x_{3}$ and $x_{4}$ and their estimates provided by the cascade observer. It illustrates the saturation of the estimates during the peaking period and the closeness of the estimates to the states beyond the peaking period. Figure $4 \mathrm{com}-$ pares the estimation errors $\tilde{x}_{3}$ and $\tilde{x}_{4}$ for the standard and high-gain observers. It illustrates that the cascade observer has convergence properties comparable to the standard one. Note that by choosing the $y$-axis to be between \pm 5 , the peaking behavior of the standard observer is truncated. Figure 5 shows the largest peaking signals in the two observers. For the standard observer $\hat{x}_{4}$ peaks to $O\left(1 / \varepsilon^{3}\right)$ value while for the cascade observer $z_{4}+\hat{x}_{3} / \varepsilon$ peaks to $O(1 / \varepsilon)$ value. Finally, Figure 6 compares the steady-state estimation errors between the two 

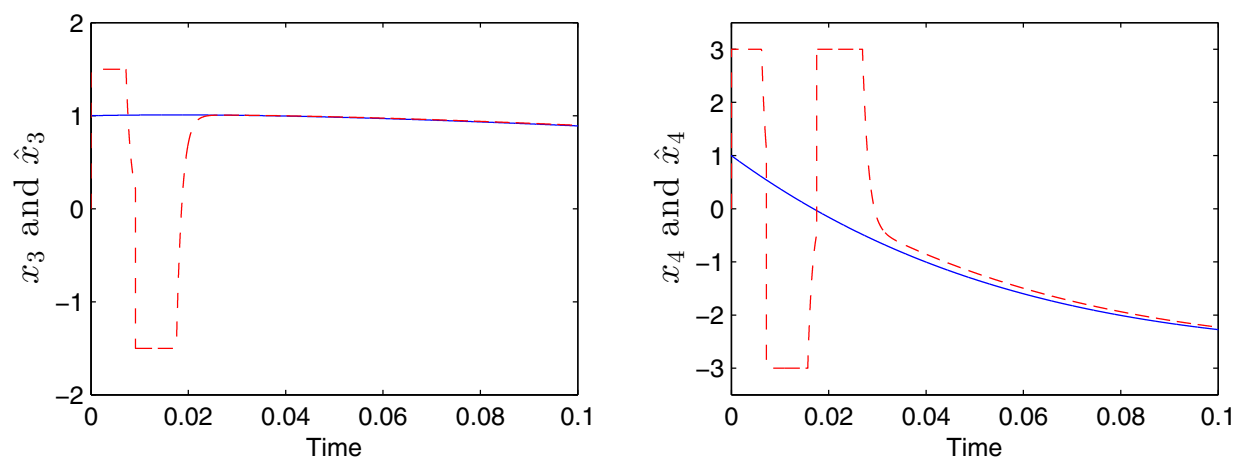

Fig. 3. Simulation of Example 1. The states $x_{3}$ and $x_{4}$ (solid) and their estimates $\hat{x}_{3}$ and $\hat{x}_{4}$ (dashed) for the cascade observer.
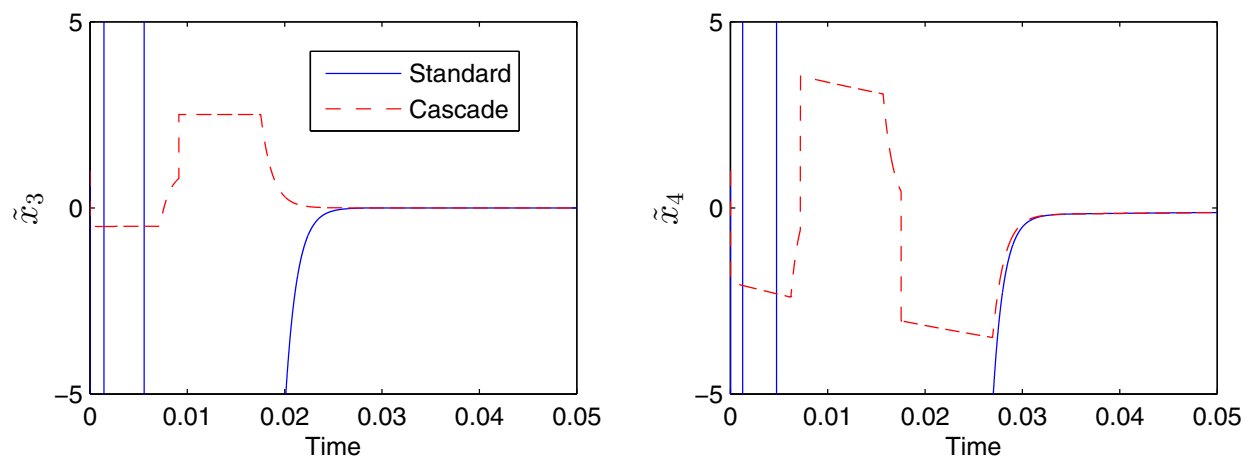

Fig. 4. Simulation of Example 1. The estimation errors $\tilde{x}_{3}$ and $\tilde{x}_{4}$ for the standard observer (solid) and the cascade observer (dashed).
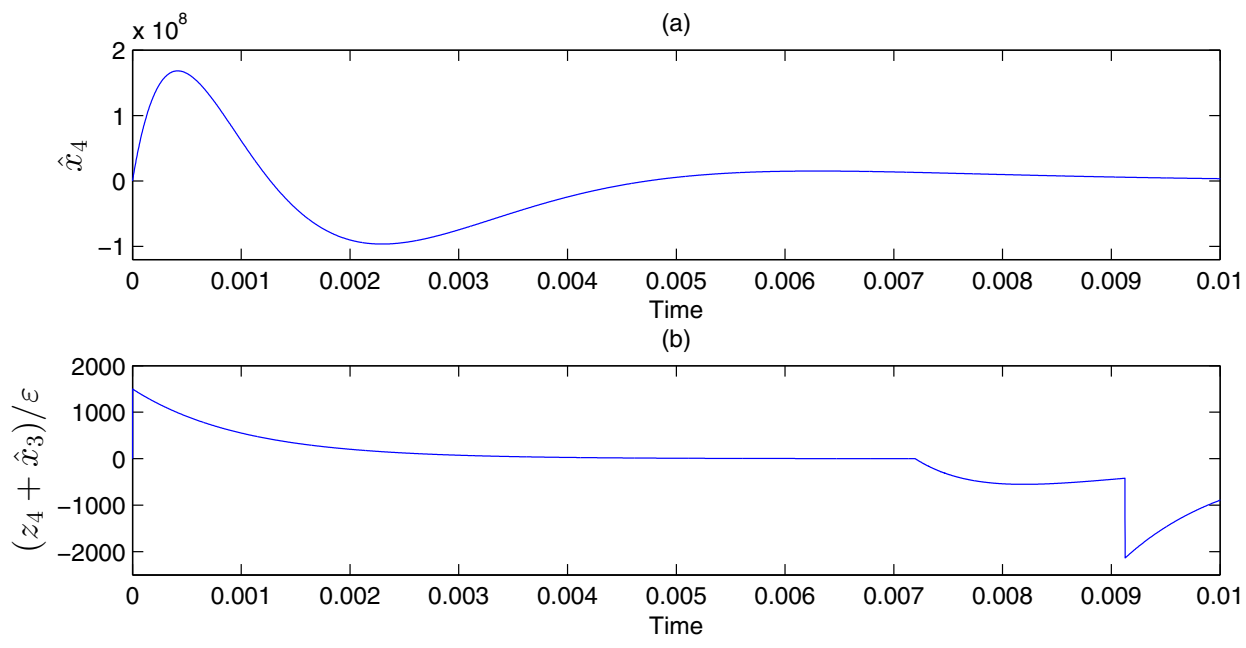

Fig. 5. Simulation of Example 1. The most peaking signal: (a) the standard observer; (b) the cascade observer.

observers, plotted in a logarithmic scale. It illustrates the fact that for $\tilde{x}_{1}, \tilde{x}_{2}$, and $\tilde{x}_{3}$ the order of the errors in the standard observer are $O\left(\varepsilon^{4}\right), O\left(\varepsilon^{3}\right)$ and $O\left(\varepsilon^{2}\right)$, respectively, compared with $O\left(\varepsilon^{2}\right), O(\varepsilon)$ and $O(\varepsilon)$ for the cascade observer.

\section{Separation Principle}

Consider the system

$$
\begin{aligned}
\triangle & =f_{0}(w, x, u) \\
\dot{x}_{i} & =x_{i+1}, \quad \text { for } 1 \leq i \leq \rho-1 \\
\dot{x}_{\rho} & =\phi(w, x, u) \\
y & =x_{1} \\
z & =f_{a}(w, x)
\end{aligned}
$$



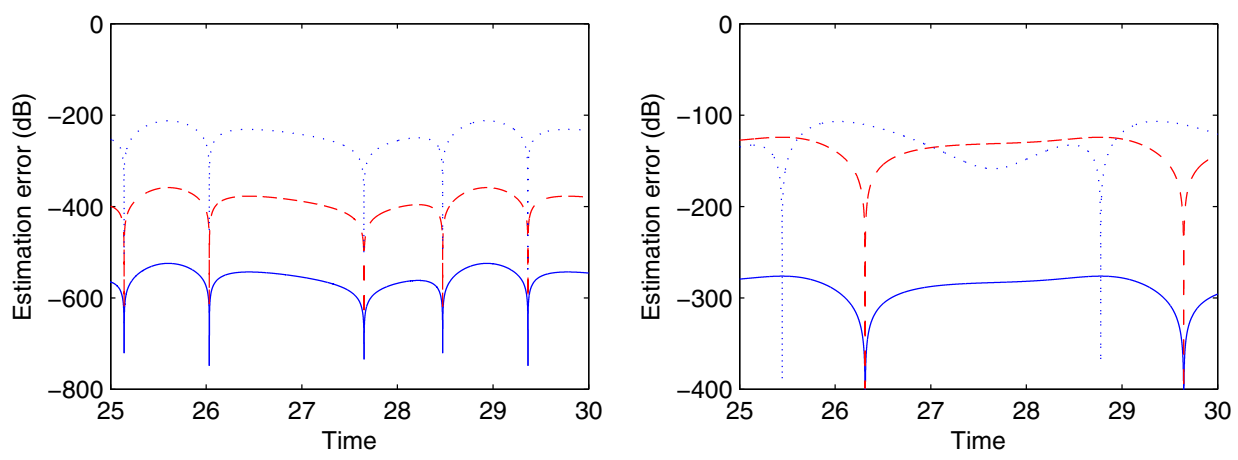

Fig. 6. The estimation errors $\tilde{x}_{1}$ (solid), $\tilde{x}_{2}$ (dashed), and $\tilde{x}_{3}$ (dotted) for the standard observer to the left and the cascade observer to the right.

where $u \in R^{m}$ is the control input, $y \in R$ and $z \in R^{s}$ are the measured outputs, and $w \in R^{\ell}$ and $x \in R^{\rho}$ constitute the state vector. The functions $f_{0}, f_{a}$, and $\phi$ are locally Lipschitz in their arguments for $(w, x, u) \in$ $D_{w} \times D_{x} \times R^{m}$, where $D_{w} \subset R^{\ell}$ and $D_{x} \subset R^{\rho}$ are domains that contain their respective origins. Moreover, $f_{0}(0,0,0)=0, f_{a}(0,0)=0$, and $\phi(0,0,0)=0$. Our goal is to design an output feedback controller to stabilize the origin.

We use a two-step approach. First, a partial state feedback controller that uses measurements of $x$ and $z$ is designed to asymptotically stabilize the origin. Then, a cascade high-gain observer is used to estimate $x$ from $y$. The state feedback controller takes the form ${ }^{3}$

$$
u=\gamma(x, z), \quad \gamma(0,0)=0
$$

where $\gamma$ is locally Lipschitz. For convenience, we write the closed-loop system under state feedback as

$$
\dot{\chi}=f(\chi)
$$

where $\chi=\operatorname{col}(w, x)$. By design, the origin of (43) is asymptotically stable. Let $V(\chi)$ be a continuously differentiable, positive definite Lyapunov function that satisfies the inequality

$$
\frac{\partial V}{\partial \chi} f(\chi) \leq-W(\chi)
$$

for all $\chi \in D_{w} \times D_{x}$, where $W(\chi)$ is a continuous positive definite function. Choose a positive constant $c$ such that $\Omega_{c}=\{V(\chi) \leq c\}$ is compact and contained in $D_{w} \times D_{x}$. Then, $\Omega_{c}$ is a positively invariant set of (43); that is, $\chi(0) \in \Omega_{c}$ implies that $\chi(t) \in \Omega_{c}$ for all $t \geq 0$. The existence of a Lyapunov function satisfying (44) follows from

\footnotetext{
3 In separation results for the standard high-gain observer [5], the state feedback control is allowed to be a dynamic controller. This extension can be allowed here as well but it is omitted for conciseness of the presentation.
}

the converse Lyapunov theorem [4, Theorem 4.17], but we need here to know $V(\chi)$. In output feedback control we saturate the state estimates and the feedback control outside $\Omega_{c}$. Towards that end, let $M_{i}>\max _{x \in \Omega_{c}}\left|x_{i}\right|$ for $i=1, \ldots \rho$. The cascade observer (18)-(22) is implemented with $\hat{x}_{2}$ to $\hat{x}_{\rho}$ saturated at $\pm M_{2}$ to $\pm M_{\rho}$, respectively. The output feedback control is taken as

$$
u=\gamma(\hat{x}, z)
$$

Theorem 2 Consider the closed-loop system of the plant (37)-(41) and the output feedback controller (45). Let $\Omega_{a}=\{V(x) \leq a\}$, with $a<c$, and $\mathcal{Q} \mathcal{Q}$ be any compact subset of $R^{\rho}$. Then, given any $\mu>0$ there exist $\varepsilon^{*}>0$ and $T^{*}>0$, dependent on $\mu$, such that for every $0<\varepsilon \leq \varepsilon^{*}$, the solutions $(\chi(t), \hat{x}(t))$ of the closed-loop system, starting in $\Omega_{a} \times \mathcal{Q}$, are bounded for all $t \geq 0$ and satisfy

$$
\begin{gathered}
\|\chi(t)\| \leq \mu \quad \text { and } \quad\|\hat{x}(t)\| \leq \mu, \quad \forall t \geq T^{*} \\
\left\|\chi(t)-\chi_{r}(t)\right\| \leq \mu, \quad \forall t \geq 0
\end{gathered}
$$

where $\chi_{r}$ is the solution of (43), starting at $\chi(0)$. Moreover, if the origin of (43) is exponentially stable, then the origin of the closed-loop system is exponentially stable and $\Omega_{a} \times \mathcal{Q}$ is a subset of its region of attraction.

Proof: Since $\hat{x}$ is bounded, uniformly in $\varepsilon$, and $\chi(0)$ belongs to $\Omega_{a}$, where $a<c$, there exist positive constants $T_{0}$ and $b \in(a, c)$, both independent of $\varepsilon$, such that $\chi(t) \in \Omega_{b}$ for all $t \in\left[0, T_{0}\right]$. During this time interval, the conditions of Theorem 1 hold and we conclude that the estimation errors satisfy (28) and (29) for $t \geq T(\varepsilon)$, where $\lim _{\varepsilon \rightarrow 0} T(\varepsilon)=0$. Thus, there is $\varepsilon_{1}^{*}>0$ such that $T(\varepsilon)<T_{0}$ for all $\varepsilon \in\left(0, \varepsilon_{1}^{*}\right]$. The upper bounds on the estimation error imply that there is $\varepsilon_{2}^{*}>0$ such that for all $\varepsilon \in\left(0, \varepsilon_{2}^{*}\right], \hat{x}(t) \in \Omega_{c}$ and since $\hat{x}(t)$ is saturated outside $\Omega_{c}$, we see that for $t \in\left[T(\varepsilon), T_{0}\right], \hat{x}(t)$ is not saturated. Then, the $\dot{\chi}$ equation under output feedback can be written as

$$
\dot{\chi}=f_{c}(\chi, x-\hat{x})
$$


where $f_{c}(\chi, 0)=f(\chi)$. Calculating the derivative of $V(\chi)$ along the trajectories of (48) we obtain

$$
\begin{aligned}
\dot{V} & =\frac{\partial V}{\partial \chi} f(\chi)+\frac{\partial V}{\partial \chi}\left[f_{c}(\chi, x-\hat{x})-f_{c}(\chi, 0)\right] \\
& \leq-W(\chi)+k_{a}\|x-\hat{x}\| \leq-W(\chi)+k_{b} \varepsilon
\end{aligned}
$$

for some positive constants $k_{a}$ and $k_{b}$. Thus, there is $\varepsilon_{3}^{*}$ such that for all $\varepsilon \in\left(0, \varepsilon_{3}^{*}\right], \dot{V}<0$ on the boundary of $\Omega_{b}$. Hence, $\chi(t)$ never leaves $\Omega_{b}$ and the estimation errors satisfy (28) and (29) for all $t \geq 0$. Inequality (49) shows also that $\|\chi\|$ is ultimately bounded by a class $\mathcal{K}$ function of $\varepsilon$, and from (28) and (29) we see that $\|\hat{x}\|$ is also ultimately bounded by a class $\mathcal{K}$ function of $\varepsilon$. Therefore, given any $\mu>0$ we can find $\varepsilon_{4}^{*}>0$ and $T^{*}>0$ such that (46) is satisfied for $\varepsilon \in\left(0, \varepsilon_{4}^{*}\right]$.

To prove (47) we note that, since $\lim _{t \rightarrow \infty} \chi_{r}(t)=0$, given any $\mu>0$, there is $T_{1}>0$ such that $\left\|\chi_{r}(t)\right\| \leq \mu / 2$ for all $t \geq T_{1}$. On the other hand, in view of (46), there is $T_{2}>0$ such that $\|\chi(t)\| \leq \mu / 2$ for all $t \geq T_{2}$. Taking $T_{3}=\max \left\{T_{1}, T_{2}\right\}$, we see that $\left\|\chi(t)-\chi_{r}(t)\right\| \leq \mu$ for all $t \geq T_{3}$. So we are left with the task of showing (47) on the compact interval $\left[0, T_{3}\right]$. We divide $\left[0, T_{3}\right]$ into the two intervals $[0, T(\varepsilon)]$ and $\left[T(\varepsilon), T_{3}\right]$ and show (47) on each interval. Consider first $[0, T(\varepsilon)]$. Since $\gamma(\hat{x}, z)$ is bounded uniformly in $\varepsilon$, there is a positive constant $k_{c}$ such that $\|\chi(t)-\chi(0)\| \leq k_{c} t$. Similarly, there is a positive constant $k_{d}$ such that $\left\|\chi_{r}(t)-\chi_{r}(0)\right\| \leq k_{d} t$. Because $\chi(0)=\chi_{r}(0),\left\|\chi(t)-\chi_{r}(t)\right\| \leq 2 k_{e} T(\varepsilon)$ for all $t \in[0, T(\varepsilon)]$, where $k_{e}=\max \left\{k_{c}, k_{d}\right\}$. Since $T(\varepsilon) \rightarrow 0$ as $\varepsilon \rightarrow 0$, there exists $\varepsilon_{5}^{*}>0$ such that (47) holds on $[0, T(\varepsilon)]$ for every $\varepsilon \in\left(0, \varepsilon_{5}^{*}\right]$. Next, consider the interval $\left[T(\varepsilon), T_{3}\right]$. Inequality (47) follows from continuous dependence of the solutions of differential equations on initial states and right-hand side functions [4, Theorem $3.5]$ because $f_{c}(\chi, x-\hat{x})$ is $O(\varepsilon)$ close to $f_{c}(\chi, 0)=f(\chi)$ and $\left\|\chi(T(\varepsilon))-\chi_{r}(T(\varepsilon))\right\| \leq 2 k_{e} T(\varepsilon)$.

To prove exponential stability, we construct a nonminimal realization of the closed-loop system under output feedback for $t \geq T(\varepsilon)$. We have seen in the proof of Theorem 1 that $\psi_{2}=\operatorname{col}\left(\eta_{1}, \eta_{2}\right)$ satisfies equation (32) and, for $i=3, \ldots, \rho, \eta_{i}$ is the output of the system (36), where for $i=\rho, x_{\rho+1}$ is replaced by $\phi$. The change of variables

$$
\varphi_{2}=\frac{1}{\varepsilon} \psi_{2}, \quad \varphi_{i}=\frac{1}{\varepsilon^{i-1}} \tilde{\psi}_{i}, \text { for } 3 \leq i \leq \rho
$$

results in

$$
\begin{aligned}
& \varepsilon \dot{\varphi}_{2}=F_{2} \varphi_{2}+\varepsilon G_{23} x_{3} \\
& \varepsilon \dot{\varphi}_{i}=F_{i} \varphi_{i}+\varepsilon G_{i} x_{i+1}, \quad \text { for } 3 \leq i \leq \rho
\end{aligned}
$$

where $G_{i}=\sum_{k=3}^{i+1} F_{i}^{-(i-k+1)} G_{i k}$. Using (31), we conclude that $x-\hat{x}$ is the output of the system

$$
\varepsilon \dot{\varphi}=F \varphi+\varepsilon[G x+E \phi(w, x, u)], \quad x-\hat{x}=H \varphi
$$

where

$$
\begin{gathered}
\varphi=\operatorname{col}\left(\varphi_{2}, \varphi_{3}, \ldots, \varphi_{\rho}\right), F=\text { bloc-diag }\left[F_{2}, F_{3}, \ldots, F_{\rho}\right] \\
G=\left[\begin{array}{cccccc}
0 & G_{23} & 0 & 0 & \ldots & 0 \\
0 & 0 & G_{3} & 0 & \ldots & 0 \\
\vdots & & & & \vdots \\
0 & \ldots & 0 & G_{\rho-1} \\
0 & \ldots & 0 & 0
\end{array}\right], \quad E=\left[\begin{array}{c}
0 \\
\vdots \\
\vdots \\
0 \\
G_{\rho}
\end{array}\right]
\end{gathered}
$$

$H=\operatorname{bloc}-\operatorname{diag}\left[H_{2}, H_{3}, \ldots, H_{\rho}\right]$, in which $H_{2}=\left[\begin{array}{ll}\varepsilon & 0 \\ 0 & 1\end{array}\right]$

The matrix $F$ is Hurwitz because all the matrices $F_{2}$ to $F_{\rho}$ are Hurwitz. This realization is non minimal because the dimension of $\varphi$ is $2+3+4+\cdots+\rho$. The closed-loop system under output feedback can be represented by

$$
\begin{aligned}
\dot{\chi} & =f_{c}(\chi, H \varphi) \\
\varepsilon \dot{\varphi} & =F \varphi+\varepsilon\left[G x+E \phi\left(w, x, \gamma\left(x-H \varphi, f_{a}(w, x)\right)\right]\right.
\end{aligned}
$$

Assuming that the origin of $\dot{\chi}=f(\chi)$ is exponentially stable, it follows from the converse Lyapunov theorem [4, Theorem 4.14] that there is a continuously differentiable Lyapunov function $V_{1}(\chi)$, which satisfies the inequalities

$$
\begin{gathered}
b_{1}\|\chi\|^{2} \leq V_{1}(\chi) \leq b_{2}\|\chi\|^{2} \\
\frac{\partial V_{1}}{\partial \chi} f(\chi) \leq-b_{3}\|\chi\|^{2} \\
\left\|\frac{\partial V_{1}}{\partial \chi}\right\| \leq b_{4}\|\chi\|
\end{gathered}
$$

over a ball $B_{r} \subset R^{\ell+\rho}$ for some positive constants $r$ and $b_{1}$ to $b_{4}$. Consider the composite Lyapunov function $V_{2}(\chi, \varphi)=V_{1}(\chi)+\varphi^{T} P_{0} \varphi$, where $P_{0}$ is the positive definite solution of the Lyapunov equation $P_{0} F+F^{T} P_{0}=$ $-I$. Using the local Lipschitz properties of $f, f_{a}, \phi$, and $\gamma$, and the fact that $\phi, f_{a}$, and $\gamma$ vanish at the origin, it can be shown that the derivative of $V_{2}$ along the trajectories of the system (50)-(51) satisfies the inequality

$$
\dot{V}_{2} \leq-\mathcal{Y}^{T} Q_{0} \mathcal{Y}
$$

where

$$
Q_{0}=\left[\begin{array}{cc}
b_{3} & -b_{5} \\
-b_{5} & (1 / \varepsilon)-b_{6}
\end{array}\right], \quad \mathcal{Y}=\left[\begin{array}{c}
\|\chi\| \\
\|\varphi\|
\end{array}\right]
$$

for some nonnegative constants $b_{5}$ and $b_{6}$. The matrix $Q_{0}$ is positive definite for sufficiently small $\varepsilon$. Hence, there is a neighborhood $\mathcal{N}$ of the origin $(\chi=0, \varphi=0)$, independent of $\varepsilon$, and $\varepsilon_{6}^{*}>0$ such that for every $\varepsilon \in\left(0, \varepsilon_{6}^{*}\right]$, the origin is exponentially stable and every trajectory 
in $\mathcal{N}$ converges to the origin as $t \rightarrow \infty$. By (46), there is $\varepsilon_{7}^{*}>0$ such that for every $\varepsilon \in\left(0, \varepsilon_{7}^{*}\right]$ and every $(\chi(0), \hat{x}(0)) \in \Omega_{a} \times \mathcal{Q}$, the solution of $(50)-(51)$ enters $\mathcal{N}$ in finite time. Hence, for every $\varepsilon \in\left(0, \min \left\{\varepsilon_{6}^{*}, \varepsilon_{7}^{*}\right\}\right]$, the origin of the closed-loop system is exponentially stable and $\Omega_{a} \times \mathcal{Q}$ is a subset of the region of attraction.

Theorem 2 shows a number of properties of the output feedback controller when $\varepsilon$ is sufficiently small. First, it shows that the trajectories are bounded. Second, (46) shows practical stabilization because the trajectories can be brought to an arbitrarily small neighborhood of the origin by choosing $\varepsilon$ small enough. Third, (47) shows that the solution $\chi(t)$ under output feedback approaches the solution $\chi_{r}(t)$ under state feedback as $\varepsilon$ tends to zero. Fourth, the output feedback controller recovers exponential stability when the origin under state feedback is exponentially stable. Finally, If the assumptions hold globally, the origin under state feedback is globally asymptotically stable and locally exponentially stable, and the function $V(x)$ is radially unbounded, then for sufficiently small $\varepsilon$, the output feedback controller achieves semiglobal stabilization because the set $\Omega_{a}=$ $\{V(\chi) \leq a\}$ can be chosen to contain any given compact subset of $R^{\ell+\rho}$.

Theorem 2 reads almost the same as the corresponding theorem for the standard high-gain observer; see for example Theorem 12.2 of [5]. There is, however, a difference between the two cases. In the case of the standard observer we require the function $\gamma(x, z)$ to be globally bounded in $x$ but we do not require knowledge of the Lyapunov function $V(\chi)$. Global boundedness of $\gamma$ is typically achieved by saturation outside a compact set, but we do not have to explicitly describe such set. Once we know that the globally bounded $\gamma$ stabilizes the origin, we know that there is a region of attraction $\mathcal{R}$ and the initial conditions can belong to any compact subset of $\mathcal{R}$. The Lyapunov function $V(\chi)$ is established in the proof from a converse Lyapunov theorem. In the current case, the situation is different because we have to saturate the estimates $\hat{x}_{2}$ to $\hat{x}_{\rho}$ in the cascade observer, so we need to explicitly specify the compact set over which saturation is carried out. This is achieved here by explicitly requiring knowledge of the Lyapunov function $V(\chi)$ and saturation is performed outside the compact set $\Omega_{c}=\{V(\chi) \leq c\}$.

Example 2 Consider the system

$\dot{x}_{1}=x_{2}, \quad \dot{x}_{2}=x_{3}, \quad \dot{x}_{3}=x_{4}, \quad \dot{x}_{4}=\phi(x)+u, \quad y=x_{1}$

where $\phi(x)$ is the same as in Example 1. The goal is to stabilize the origin without the knowledge of $\phi$. The state feedback is designed using Lyapunov redesign [5, Section 10.2]. Rewrite the state equation as $\dot{x}=A x+$ $B[\phi(x)+u]$, where the pair $(A, B)$ represents a chain of four integrators. The matrix $K=\left[\begin{array}{llll}1 & 4 & 6 & 4\end{array}\right]$ assigns all the eigenvalues of $A-B K$ at -1 . By adding and subtracting $K x$, we rewrite the state equation as $\dot{x}=$ $(A-B K) x+B[u+\delta(x)]$, where

$\delta(x)=\phi(x)+K x=\left[3-\left(x_{1}+2 x_{2}+x_{3}\right)^{2}\right]\left(x_{2}+2 x_{3}+x_{4}\right)$

Let $P=P^{T}>0$ be the solution of the Lyapunov equation $P(A-B K)+(A-B K)^{T} P=-Q$, with $Q=$ $\operatorname{diag}[0.1,0.1,1,1] ; V(x)=x^{T} P x$ is a Lyapunov function for the linear system $\dot{x}=(A-B K) x$. According to the Lyapunov redesign procedure, a stabilizing state feedback control can be taken as

$$
u=-\beta \operatorname{sat}\left(\frac{2 \beta B^{T} P x}{\mu}\right)
$$

where $\beta$ is an upper bound on $|\delta(x)|$ over the compact set of interest and $\mu$ is a positive constant. Suppose all initial states of interest are in the interior of $\Omega=\{V(x) \leq 1.2\}$ and calculate an upper bound $|\delta(x)|$. Using the fact that $\max _{x^{T} P x \leq c}\left|b^{T} x\right|=\sqrt{c}\left\|b^{T} P^{-1 / 2}\right\|[5$, Appendix B], it can be shown that $\left|x_{1}\right| \leq 2.7397,\left|x_{2}\right| \leq 1.4881$, $\left|x_{3}\right| \leq 1.0747,\left|x_{4}\right| \leq 2.7337,\left|x_{1}+2 x_{2}+x_{3}\right|^{2} \leq 3.6085$, $\left|3-\left(x_{1}+2 x_{2}+x_{3}\right)^{2}\right| \leq 3$, and $\left|x_{2}+2 x_{3}+x_{4}\right| \leq 2.5595$ over $\Omega$. From the last two bounds, it can be shown that $|\delta(x)| \leq 7.6785$ over $\Omega$. We take $\beta=12$. Near the origin, $|\delta(x)| \leq 3\left|x_{2}+2 x_{3}+x_{4}\right| \varrho_{1}\|x\|$, where $\varrho_{1}=7.35$. Application of Theorem 10.4 of [5] shows that the origin is asymptotically stable for $\mu<4 \times 0.9 \times\left(\beta / \varrho_{1}\right)^{2}=9.956$. We take $\mu=1$. Thus, the state feedback control is given by

$u=-12$ sat $\left(1.2 x_{1}+4.05 x_{2}+7.8 x_{3}+4.95 x_{4}\right) \stackrel{\text { def }}{=} \gamma(x)$

and the output feedback control is taken as $u=\gamma(\hat{x})$. In the cascade observer, $\hat{x}_{2}, \hat{x}_{3}$, and $\hat{x}_{4}$ are saturated at $\pm 1.6, \pm 1.2$, and \pm 3 , respectively. The simulations of Figures 7 and 8 are carried out with $x(0)=\operatorname{col}(1,0,0,-2)$ and zero initial conditions for the observers. Note that $x(0)$ is in the interior of $\Omega$. Figure 7 shows the state trajectories under state and output feedback when the cascade observer is used. It demonstrates that the trajectories under output feedback approach the ones under state feedback as $\varepsilon$ decreases. Figure 8 compares the performance of the standard and cascade observers for $\varepsilon=0.001$. Both observers are taken from Example 1. It shows that the performance of the cascade observer is comparable to the performance of the standard observer.

\section{Conclusions}

A new cascade high-gain observer is constructed by cascading a second-order observer with a series of first-order ones, and inserting saturation functions in between the 

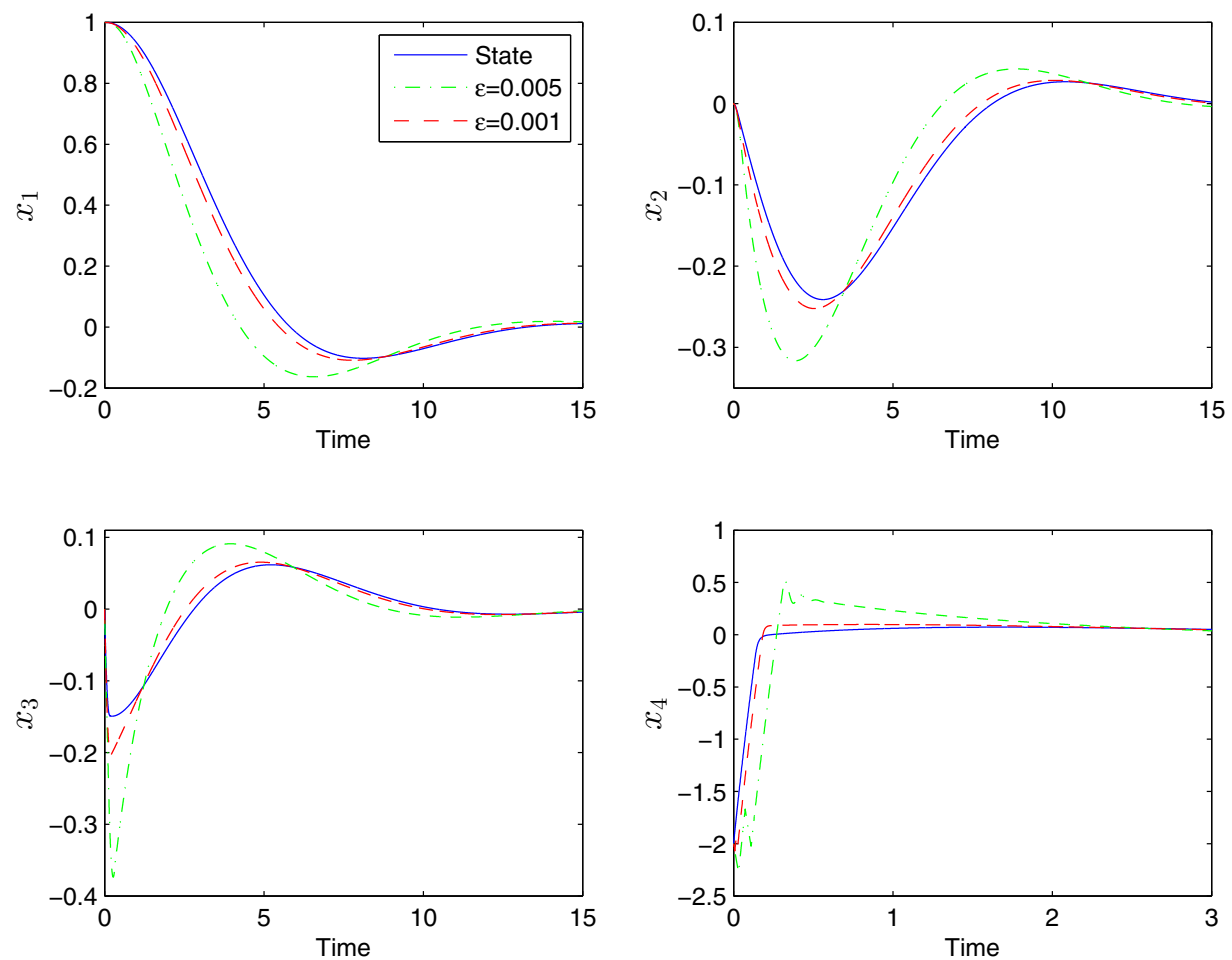

Fig. 7. Simulation of Example 2. The state trajectories under state feedback (solid) and output feedback with the cascade observer for $\varepsilon=0.005$ (dash-dotted) and $\varepsilon=0.001$ (dashed).
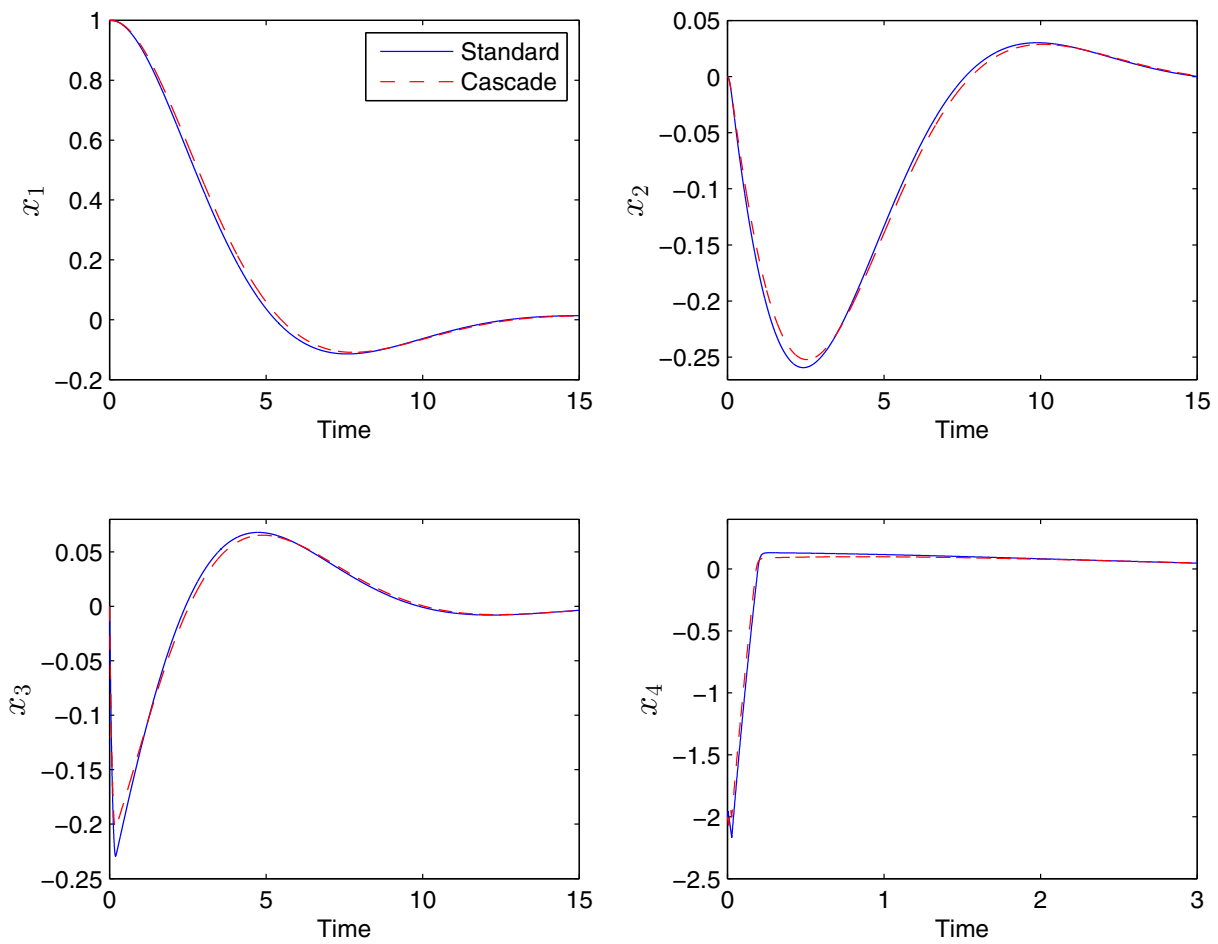

Fig. 8. Simulation of Example 2. The state trajectories under output feedback with the standard (solid) and cascade (dashed) observers for $\varepsilon=0.001$. 
different stages. The observer has a numerical advantage over the standard high-gain observer because its gain is of the order of $1 / \varepsilon$, compared with $1 / \varepsilon^{\rho}$ for the standard observer, and its peaking signal is of the order of $1 / \varepsilon$, compared with $1 / \varepsilon^{\rho-1}$. Theoretical results similar to those of the standard observer are proved for the new observer and simulation shows that its performance in feedback control is comparable to the performance of the standard observer.

It is worthwhile to mention that the effect of measurement noise on a feedback control system that uses the cascade observer is comparable to its effect on a system that uses the standard observer; in other words, it is not better or worse. It can be shown that $\|x(t)-\hat{x}(t)\|$ has the same order of magnitude, $O\left(\mu / \varepsilon^{\rho-1}\right)$, in both observers where $\mu$ is a bound on the measurement noise. Also, when the estimates are not saturated, the transfer function from the measurement noise to the control signal under the cascade observer has the same frequencyresponse of the transfer function under a standard linear observer.

\section{References}

[1] D. Astolfi and L. Marconi. A high-gain nonlinear observer with limited gain power. IEEE Trans. Automat. Contr., 60:30593064, 2015.

[2] D. Astolfi, L. Marconi, and A. Teel. Lower-power peaking-free high-gain observers for nonlinear systems. In Proc. European Control Conf., Aablorg, Denmark, 2016.

[3] A.N. Atassi and H.K. Khalil. A separation principle for the stabilization of a class of nonlinear systems. IEEE Trans. Automat. Contr., 44:1672-1687, 1999.

[4] H.K. Khalil. Nonlinear Systems. Prentice Hall, Upper Saddle River, New Jersey, 3rd edition, 2002.

[5] H.K. Khalil. Nonlinear Control. Pearson, Boston, 2015.

[6] H.K. Khalil and L. Praly. High-gain observers in nonlinear feedback control. Int. J. Robust and Nonlinear Control, 24:993-1015, 2014.

[7] M. Maggiore and K. Passino. A separation principle for nonUCO systems. IEEE Trans. Automat. Contr., 48:1122-1133, 2003.

[8] A. Teel. Further variants of the Astolfi/Marconi high-gain observer. In Proc. American Control Conf., Boston, MA, 2016.

[9] A. Teel and L. Praly. Global stabilizability and observability imply semi-global stabilizability by output feedback. Syst. Contr. Lett., 22:313-325, 1994. 